\title{
Avaliaçáo da qualidade e quantidade do sono em pacientes renais crônicos submetidos à hemodiálise
}

\author{
Evaluation of the quality and quantity of sleep in chronic renal failure patients on hemodialysis
}

\section{Camila Kelly Diniz Guimarães ${ }^{1}$, Débora Almeida Galdino Alves², Laiz Helena de Castro Toledo Guimarães ${ }^{3}$}

\begin{abstract}
RESUMO
Objetivo. O objetivo deste estudo foi avaliar a qualidade e a quantidade do sono em pacientes renais crônicos submetidos à hemodiálise na Santa Casa de Misericórdia de Lavras - Minas Gerais, Brasil. Método. Participaram do estudo 40 pacientes, com idade entre 40 e 60 anos. Foi utilizado o Diário do Sono para avaliar a quantidade e o Índice de Qualidade de Sono de Pittsburgh para avaliar a qualidade do sono dos pacientes. Resultados. De acordo com a análise descritiva do Diário do Sono, a média do tempo total de sono foi de 6,2 h $( \pm 1,3)$, a média do número de cochilos por dia foi de $0,4( \pm 0,5)$ e os despertares noturnos tiveram uma média de $0,9( \pm 1,0)$. A análise do Índice de Qualidade de Sono de Pittsburgh mostrou que 20\% dos pacientes apresentaram boa qualidade de sono, 52,5\% apresentaram qualidade de sono ruim e $27,5 \%$ apresentaram distúrbios do sono. Conclusáo. Concluiu-se que a maioria dos pacientes do estudo apresentou, de acordo com o Diário do Sono, quantidade de sono abaixo do satisfatório e de acordo com o Índice de Qualidade de Sono de Pittsburgh, qualidade de sono ruim ou distúrbios do sono.
\end{abstract}

Unitermos. Sono, Hemodiálise, Insuficiência Renal Crônica.

Citação. Guimarães CKD, Alves DAG, Guimarães LHCT. Avaliação da qualidade e quantidade do sono em pacientes renais crônicos submetidos à hemodiálise.

\begin{abstract}
Objective. The purpose of this paper was to evaluate quality and quantity of sleep of chronic renal patients undergoing hemodialysis in Santa Casa da Misericórdia Hospital in Lavras - Minas Gerais, Brazil. Method. Forty patients between 40 and 60 years old took part in the study. The Diary of Sleep was used to evaluate the quantity and The Pittsburgh Sleep Quality Index to evaluate the quality of sleep in those patients. Results. According to the descriptive analysis of The Diary of Sleep, the average of the total time of sleep was $6.2 \mathrm{~h}( \pm 1,3)$, the average of the number of naps taken per day was $0.4( \pm 0,5)$ and the night awakenings had the average of $0.9( \pm 1,0)$. The analysis of The Pittsburgh Sleep Quality Index showed that 20\% of the patients displayed good quality of sleep, $52.5 \%$ displayed bad quality of sleep and $27.5 \%$ displayed sleep disorders. Conclusion. The conclusion that follows from this study is that the majority of patients presented, according to The Diary of Sleep, less than satisfactory sleep quantity and according to The Pittsburgh Sleep Quality Index, bad sleep quality or sleep disorders.
\end{abstract}

Keywords. Sleep, Hemodialysis, Chronic Renal Insufficienty.

Citation. Guimarães CKD, Alves DAG, Guimarães LHCT. Evaluation of the quality and quantity of sleep in chronic renal failure patients on hemodialysis.
Suporte Financeiro: FAPEMIG.

Trabalho realizado no UNILAVRAS, Lavras, Lavras-MG, Brasil.

1. Fisioterapeuta, Lavras-MG, Brasil.

2. Fisioterapeuta, mestre, professora do Centro Universitário de Lavras, LavrasMG, Brasil.

3. fisioterapeuta, mestre, professora doCentro Universitário de Lavras, LavrasMG, Brasil.
Endereço para correspondência: Camila K Diniz Guimarães Rua Cabo Frio, 173 - Condomínio Náutico Porto da Pedra CEP 37205-000, Ijaci-MG, Brasil.

E-mail: cacak10@hotmail.com

Original

Recebido em: 19/05/10

Aceito em: 10/03/11

Conflito de interesses: não 


\section{INTRODUÇÃO}

A doença renal crônica constitui hoje, um importante problema na saúde pública. No Brasil, o número de pacientes com insuficiência renal crônica mantidos em tratamento, dobrou nos últimos anos ${ }^{1}$. A insuficiência renal crônica é o estado resultante de uma deterioração significativa e permanente de néfrons funcionantes. É uma via final comum de várias afecçôes renais, como diabetes mellitus, hipertensão e doenças glomerulares, que se constitui como importante causa de morbidade e mortalidade ${ }^{2}$.

As alterações na vida dos pacientes renais crônicos são, particularmente, incômodas e contínuas para eles, uma vez que podem se sentir diferentes e excluídos por serem proibidos de comer certos alimentos, terem uma ingesta hídrica reduzida e controlada, necessitarem de remédios continuamente e serem submetidos ao tratamento dialítico para manutenção de suas vidas ${ }^{1}$.

Os tratamentos disponíveis nas doenças renais terminais são: a diálise peritoneal ambulatorial contínua, diálise peritoneal automatizada, diálise peritoneal intermitente, hemodiálise e transplante renal. Esses tratamentos substituem parcialmente a função renal, através do hemodialisador, aliviam os sintomas da doença e preservam a vida do paciente, porém, nenhum deles é curativo ${ }^{3}$. O hemodialisador é um filtro com uma membrana semipermeável que possui uma grande área de superfície de troca, sendo o sangue perfundido por um lado e o fluido de diálise pelo outro. Solutos em que a concentração é maior no sangue do que no fluido de diálise se difundirão através de gradiente de concentração, do sangue para o fluido ${ }^{4}$.

Os principais estressores relacionados ao paciente em tratamento hemodialítico são a restrição de líquidos e alimentos, câimbras musculares, incerteza sobre o futuro, interferências no trabalho, mudanças na estrutura familiar, medo de ficar sozinho e distúrbios do sono 5 . Durante o sono ocorrem variaçóes no volume de urina e na excreção de sódio, potássio e cálcio no sentido de redução. As mudanças do nível hormonal antidiurético são responsáveis pelas alteraçóes relacionadas ao sono na função renal ${ }^{6}$.

Alterações no sono estão presentes em até $70 \%$ dos pacientes renais crônicos ${ }^{7}$. Distúrbios do sono são uma das complicaçôes enfrentadas pelos portadores de insu- ficiência renal crônica ${ }^{5,7}$. Através de métodos diagnósticos de enfermagem, um estudo com 20 pacientes adultos em tratamento hemodialítico, encontrou padrão de sono perturbado em $80 \%$ dos pacientes $^{8}$. A má qualidade do sono, sonolência diurna excessiva e síndrome das pernas inquietas são frequentes nos pacientes em tratamento hemodialítico, porém não apresentam relação com o turno da diálise?.

Com o intuito de compreender melhor o sono nessa classe de pacientes, delineou-se o objetivo deste estudo, que foi avaliar a qualidade e a quantidade do sono em pacientes renais crônicos submetidos à hemodiálise na Santa Casa de Misericórdia de Lavras - MG.

\section{MÉTODO}

\section{Amostra}

O estudo realizado foi do tipo transversal de prevalência. Foram incluídos no estudo voluntários com idade entre 40 e 60 anos que estivessem em tratamento hemodialítico na Santa Casa de Misericórdia de Lavras, Minas Gerais, Brasil. Foram excluídos aqueles voluntários que, por qualquer motivo, deixassem de preencher a um dos questionários aplicados.

A pesquisa foi aprovada pelo Comitê de Ética em Pesquisa do UNILAVRAS dia 4 de fevereiro de 2009 com o protocolo 0140. 0. 189. 000-08 e os participantes assinaram um termo de consentimento livre e esclarecido.

\section{Procedimento}

Foram utilizados dois questionários como forma de avaliação da amostra, o Diário do Sono ${ }^{10}$ e o Índice de qualidade de sono de Pittsburgh, elaborado em 1989 por Buysse DJ e validado para a Língua portuguesa ${ }^{11}$. Dos 135 pacientes em tratamento no setor de hemodiálise da Santa Casa de Misericórdia de Lavras, 40 aceitaram responder o Pittsburgh Sleep Quality Index e desses, apenas 17 pacientes aceitaram preencher o Diário do Sono.

O Diário do Sono avaliou a quantidade de sono durante 30 dias, registrando-se diariamente quanto tempo o paciente dormiu, quanto tempo cochilou durante o dia, quantas vezes e por quanto tempo despertou durante a noite. O Diário do Sono foi explicado detalhadamente e individualmente aos voluntários e foi entregue para ser preenchido em casa. Terminado o período de preenchi- 
mento, os questionários foram recolhidos e analisados.

O Índice de Qualidade de Sono de Pittsburgh (PSQI-BR) foi utilizado para avaliar a qualidade subjetiva do sono. Este questionário é composto por 19 itens agrupados em sete componentes, cada qual pontuado em uma escala de 0 a 3. Os componentes são, respectivamente: (1) a qualidade subjetiva do sono; (2) a latência do sono; (3) a duraçâo do sono; (4) a eficiência habitual do sono; (5) as alteraçôes do sono; (6) o uso de medicações para o sono; e (7) a disfunção diurna. Os escores dos sete componentes são somados para conferir uma pontuação global do PSQI-BR, a qual varia de 0 a 21 . Pontuaçóes de $0-4$ indicam boa qualidade do sono, de 5-10 indicam qualidade ruim e acima de 10 pontos indicam distúrbio do sono. O PSQIBR foi aplicado pela autora do estudo durante a sessão de hemodiálise e, posteriormente, foi analisado ${ }^{11}$.

\section{Análise Estatística}

Foram realizadas análises descritivas como, frequências, médias e desvios padróes.

\section{RESULTADOS}

Dos 17 voluntários que preencheram o Diário do Sono $41,2 \%$ eram do sexo masculino e $58,8 \%$ do sexo feminino. $58,8 \%$ tinham idade entre 40 e 50 anos e $41,2 \%$ tinham idade entre 51 e 60 anos.

De acordo com o Diário do Sono a média do Tempo Total de Sono (TTS) foi de 6,2 $\pm 1,3$ horas. O número de cochilos resultou em uma média de $0,4 \pm 0,5$ e o número de despertares noturnos (DN) em uma média de $0,9 \pm 1,0$.

Dos 40 voluntários que responderam o PSQI-BR $40 \%$ eram do sexo masculino e $60 \%$ do sexo feminino. $52,5 \%$ tinham idade entre 40 e 50 anos e $47,5 \%$ tinham idade entre 51 e 60 anos.

Os percentuais dos escores obtidos através do PSQI-BR, relacionados à qualidade do sono da população, estão representados na Figura 1. 20\% da amostra demonstraram boa qualidade de sono; $52,5 \%$ apresentaram qualidade de sono ruim e $27,5 \%$ apresentaram distúrbios do sono.

A relação do tempo de hemodiálise com a qualidade do sono dos voluntários, obtida por meio do PSQIBR demonstrou que:
- dentre os pacientes em tratamento hemodialítico há menos de 1 ano $(n=6), 17 \%$ apresentaram boa qualidade de sono, $66 \%$ apresentaram qualidade de sono ruim e 17\% apresentaram distúrbios do sono;

- dentre os pacientes em tratamento hemodialítico entre 1 e 2,9 anos ( $\mathrm{n}=7), 14 \%$ apresentaram boa qualidade de sono, $57 \%$ apresentaram qualidade de sono ruim e $29 \%$ apresentaram distúrbios do sono;

- dentre os pacientes em tratamento hemodialítico entre 3 e 4,9 anos $(n=12)$, 33\% apresentaram boa qualidade de sono, $42 \%$ apresentaram qualidade de sono ruim e $25 \%$ apresentaram distúrbios do sono;

- dentre os pacientes em tratamento hemodialítico entre 5 e 6,9 anos ( $n=4), 0 \%$ apresentou boa qualidade de sono, $75 \%$ apresentaram qualidade de sono ruim e $25 \%$ apresentaram distúrbios do sono;

- dentre os pacientes em tratamento hemodialítico entre 7 e 8,9 anos ( $n=6), 17 \%$ apresentaram boa qualidade de sono, $33 \%$ apresentaram qualidade de sono ruim e $50 \%$ apresentaram distúrbios do sono;

- dentre os pacientes em tratamento hemodialítico há 9 anos ou mais $(\mathrm{n}=5), 20 \%$ apresentaram boa qualidade de sono, $60 \%$ apresentaram qualidade de sono ruim e $20 \%$ apresentaram distúrbios do sono.

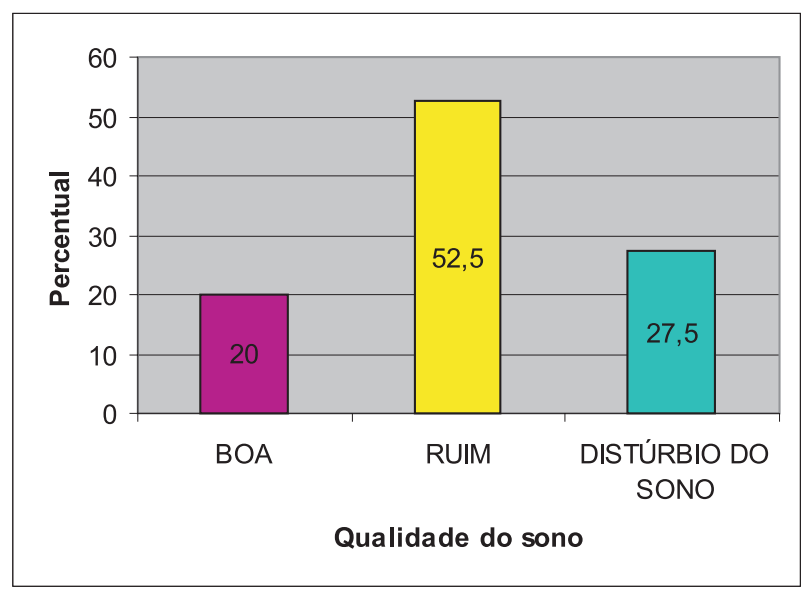

Figura 1. Qualidade do sono da amostra obtida através do Índice de Qualidade de Sono de Pittsburgh.

\section{DISCUSSÃO}

No presente estudo, a maioria dos pacientes em tratamento hemodialítico eram do sexo feminino, diferente de um outro estudo ${ }^{8}$, em que a maioria dos pacien- 
tes de hemodiálise eram do sexo masculino.

Quanto à idade, os participantes apresentaram idade entre 40 e 60 anos. Outro estudo também utilizou essa faixa etária em sua pesquisa, pois constitui um maior número de sujeitos no programa de hemodiálise ${ }^{12}$.

Com relação ao tempo de tratamento hemodialítico, outros estudos não encontraram resultados estatisticamente significantes ao relacionarem o tempo de tratamento com a qualidade do sono ${ }^{3}$. Concordando com tais achados, no atual estudo, a qualidade do sono dos pacientes, avaliados através do PSQI-BR, não obteve um aspecto linear com o tempo de tratamento. O grupo em tratamento entre 7 e 8,9 anos apresentou maior porcentagem de pacientes com distúrbios do sono. Náo foram encontrados estudos que justificassem estes achados.

Foi observado que $80 \%$ dos pacientes deste estudo apresentaram, de acordo com o PSQI-BR, qualidade do sono ruim ou distúrbio do sono. Apenas $20 \%$ da amostra do estudo apresentaram uma boa qualidade de sono. Estes achados corroboram com outros estudos ${ }^{3,6,8,9,12}$, que observaram deficiência no padrão de sono de pacientes submetidos à hemodiálise.

Com relação à análise do Diário do Sono, o tempo total de sono (TTS) consistiu no período entre o horário que o paciente dormiu à noite até o horário que ele acordou de manhá. Sabe-se que para se obter um estado ótimo de vigília, o adulto requer uma média de 7 a 8 horas de sono por $\mathrm{dia}^{13}$, o que demonstra que a média dos voluntários deste estudo para TTS, que foi de $6,2 \mathrm{~h}( \pm$ $1,3)$, ficou abaixo do ideal. Normalmente os cochilos têm duração de 15 a 60 minutos, podendo ocorrer várias vezes ao dia, contudo, quando aumentados em quantidade ou duração, podem interferir no sono noturno ${ }^{13}$. Os despertares noturnos dos pacientes que preencheram o Diário do Sono alcançaram uma média de $0,9( \pm 1,0)$. Em geral, os despertares noturnos não deveriam ultrapassar 5\% do tempo total na cama ${ }^{14}$.

$\mathrm{O}$ número de pacientes que aceitou preencher o Diário foi pequeno. Dos 135 pacientes de hemodiálise, somente 17 preencheram, fazendo-se necessária uma pesquisa com uma amostra maior. Dos pacientes que não preencheram o Diário do Sono, exceto os que se enquadraram nos critérios de exclusão, a maioria considerou que o preenchimento seria uma preocupação a mais em seu dia a dia. Vale ressaltar o excesso de compromissos clínicos que essa classe de pacientes já cumpre rotineiramente. Outro aspecto que justificou a falta de motivação para participar da pesquisa foi o comprometimento emocional dos hemodialíticos, o que pode ter sido determinante para a baixa adesão dos pacientes ao estudo.

Um aspecto relevante desse estudo foi que nenhum outro havia avaliado o sono de pacientes em tratamento hemodialítico, através do Diário do Sono e do PSQI-BR. Ressalta-se que este estudo foi o primeiro a utilizar o Diário do Sono para avaliar o sono destes pacientes.

\section{CONCLUSÃO}

Concluiu-se que a qualidade do sono de $80 \%$ dos participantes desse estudo classificou-se como ruim ou distúrbios do sono. Com relação à média de sono da amostra, os pacientes apresentaram quantidade de sono abaixo do satisfatório.

\section{REFERÊNCIAS}

1.Queiroz MVO, Dantas MCQ, Ramos IC, Jorge MSB. Tecnologia do cuidado ao paciente renal crônico: enfoque educativo-terapêutico a partir das necessidades dos sujeitos. Texto Contexto Enferm 2008;17:55-63.

2.Bevilacqua F, Bensoussan E, Jansen JM, Castro FS. Fisiopatologia Clínica. Rio de Janeiro: Atheneu, 1998, 660p.

3.Martins MRI, Cesarino CB. Qualidade de vida de pessoas com doença renal crônica em tratamento hemodialítico. Rev Latino-am Enfermagem 2005;13:670-6.

http://dx.doi.org/10.1590/S0104-11692005000500010

4.Soares CB, Ochiro EY, Sannomiga NT. Relação da temperatura da solução de diálise e a hipotensão arterial sintomática observada durante sessões de hemodiálise em pacientes com insuficiência renal crônica. Rev Esc de Enferm USP 2001;35:346-53.

5.Bertolin DC, Pace AE, Kusumota L, Ribeiro RCHM. Modos de enfrentamento dos estressores de pessoas em tratamento hemodialítico: revisão integrativa da literatura. Acta Paul Enferm 2008;21:179-86.

http://dx.doi.org/10.1590/S0103-21002008000500008

6.Souza EF, De Martino MMF, Lopes MHBM. Diagnósticos de enfermagem em pacientes com tratamento hemodialítico utilizando o modelo teórico de Imogene King. Rev Esc Enferm USP 2007;41:629-35.

7.Elias RM. Distúrbios do sistema nervoso central e periférico. J Bras nefrol 2004;26:40-1.

8.Lata AGB, Albuquerque JG, Carvalho LASBP, Lira ALBC. Diagnósticos de enfermagem em adultos em tratamento de hemodiálise. Acta Paul Enferm 2008;21:160-3.

http://dx.doi.org/10.1590/S0103-21002008000500004

9.Bastos JPC, Sousa RB, Nepomuceno LAM, Gutierrez-Adrianzen AO, Bruin PFC, Araújo MLLB, et al. Sleep disturbances in patients on mainte- 
nance hemodialysis: role of dialysis shift. Rev Assoc Med Bras 2007;53:492-6. http://dx.doi.org/10.1590/S0104-42302007000600014

10.Guimarães LHCT, Lima MD, Souza JA. Atividade física em grupo melhora o sono de idosas sedentárias. Rev Neurocienc 2007;15:203-6.

11.Bertolazi AN. Traduçấo, adaptação cultural e validação de dois instrumentos de avaliação do sono: escala de sonolência de Epworth e índice de qualidade de sono de Pittsburgh (dissertação). Porto Alegre: UFRGS, 2008, 93p.
12.Higa K, Kost MT, Soares DM, Morais MC, Polins BRG. Qualidade de vida de pacientes portadores de insuficiência renal crônica em tratamento de hemodiálise. Acta Paul Enferm 2008;21:203-6.

http://dx.doi.org/10.1590/S0103-21002008000500012

13.Fernandes RMF. O sono normal. Medicina 2006;39:157-68.

14.Geib LTC, Cataldo Neto A, Wainberg R, Nunes ML. Sono e envelhecimento. R. Psiquiatr 2003;25:453-65. 